\title{
Effect of a 6-Week Yoga Intervention on Swing Mechanics during the Golf Swing: A Feasibility Study
}

Graeme G.Sorbie

Chris Low

Ashley K. Richardson

This is an Accepted Manuscript of an article published by Taylor \& Francis in the International Journal of Performance Analysis in Sport, 2019, available online at: https://doi.org/10.1080/24748668.2019.1566845 
1 Effect of a 6-Week Yoga Intervention on Swing Mechanics during the Golf Swing: A

2 Feasibility Study

3

4 Running head: The influence of a yoga training programme on golf swing mechanics.

5

6 Location of study: Abertay University Biomechanics Laboratory

7

8

Graeme G. Sorbie, ${ }^{1}$ Chris. Low, ${ }^{1}$ and Ashley K. Richardson ${ }^{1}$

9

${ }^{1}$ School of Social \& Health Sciences, Sport and Exercise, Abertay University, United 10 Kingdom.

Address correspondence to Dr. Graeme Sorbie, School of Social \& Health Sciences, Sport and Exercise, Abertay University, United Kingdom; Dundee, DD1 1HG, Email: g.sorbie@abertay.ac.uk, Tel No: +44 (0)1382 308015, ORCID ID: 0000-0002-3362-267X.

Funding: No external funding received.

Conflict of Interest Disclosure: The authors have no conflict of interest to disclose. 
Recent evidence suggests that participating in physical conditioning programmes can improve golf performance, however, the effectiveness of a yoga intervention has yet to be investigated. The aim of the current study was to investigate the effectiveness of a six-week yoga intervention on golf swing mechanics. Ten male golfers participated in the laboratory-basedstudy. Golf swing mechanics were collected from two testing sessions, before and after the sixweek yoga intervention, using the Vicon motion capture system. Following the six-week yoga intervention, significant changes were observed between the yoga and control group in XFactor $(P \leq 0.05)$ and a medium effect $(d \geq 0.50)$ observed. No significant changes $(P>0.05)$ and no effect $(d<0.20)$ were observed in the X-Factor stretch. Significant changes $(P \leq 0.05)$ and a medium effect $(\mathrm{d}>0.50)$ were observed for the pelvis rotations following the yoga intervention, however, no differences were observed in torso rotations or hand velocities $(\mathrm{P}>$ 0.05). The findings of this feasibility study suggest that yoga may be a promising intervention in helping to improve golf swing mechanics, however, future research is required to confirm the effect of the use of yoga during the golf swing due to the sample size.

Key words: Biomechanics, Flexibility, Movement, ROM, Motion Analysis, Performance 
Flexibility and mobility are vital for optimal performance when performing skills in various sports (Cools et al., 2010; Sell, Tsai, Smoliga, Myers, \& Lephart, 2007; Young, Clothier, Otago, \& Liddell, 2004). In order to perform the golf swing proficiently, a golfer must display a good level of flexibility and mobility in order to maximise long game performance (Joyce, 2016), therefore, these attributes are more commonly found in lower handicap or professional golfers (Joyce, 2016; Tilley \& Macfarlane, 2012). It has been previously reported that an increase in range of motion (ROM) around the shoulder, pelvis and torso regions can enable greater rotation during the backswing, which can increase angular velocity (Chettle \& Neal, 2001) and, in turn, increase club head velocity during the downswing (Keogh \& Hume, 2012). Furthermore, displaying a good level of flexibility during the golf swing can be an important factor when aiming to improve posture during set-up and prevent injury caused by excessive al., 2003) which can have negative effects on performance (Joyce, 2016). stress on joints (Farrally et al., 2003). The hips, torso and shoulders are common sites of inflexibility in golfers, as the set-up position renders a repetitive bent over posture (Farrally et

The separation of the hips (pelvic region) and torso (thorax region) at the top of the backswing is a key component when aiming to maximise distance during the golf swing (Joyce, 2016), and has been the focus of many golf performance and injury prevention studies in recent years (Cole \& Grimshaw, 2008a, 2008b; Henry, Berglund, Millar, \& Locke, 2015; Joyce, 2016; Myers et al., 2008; Sell et al., 2007; Sorbie, Gu, Baker, \& Ugbolue, 2018). The separation of the hips and torso areas during the golf swing is known as the X-Factor (Joyce, 2016). In addition to the $\mathrm{X}$-Factor, the $\mathrm{X}$-Factor stretch is becoming increasingly popular within golf scientific research (An, Wulf, \& Kim, 2013; Cheetham, Martin, Mottram, \& St. Laurent, 2001; Henry et al., 2015; Sorbie et al., 2018). The X-Factor stretch refers to the additional rotation 
that occurs between the hips and torso areas in the early stage of the downswing (Sorbie et al., 2018). In order to increase the X-Factor at the top of the backswing and display an X-Factor stretch during the initiation of the downswing, a golfer must display a good level of flexibility around the pelvis and torso regions (Joyce, 2016). Greater X-Factor angles are often attributed to lower handicap and elite golfers (Joyce, 2016). For example, Cole \& Grimshaw, (2008b) found a significant difference in the X-Factor between low handicap $(<10)$ golfers $(61.4 \pm 10.8$ $\left.{ }^{\circ}\right)$ and high handicap (>18) golfers $\left(54.1 \pm 15.0^{\circ}\right)$. Zheng et al., (2008) found similar significant differences in the X-Factor angle when testing PGA Tour players $\left(56.0 \pm 4.0^{\circ}\right)$ and high handicap golfers $\left(48.0 \pm 2.0^{\circ}\right)$.

3

In addition to reduced skill level, an individual's physical limitations, such as muscular strength and flexibility levels, have a strong influence on the effectiveness of the X-Factor and X-Factor stretch (Hellström, 2009), and this ultimately has a negative effect on driving performance (Joyce, 2016). In order to improve these attributes, golf specific strength and conditioning programmes are becoming prominent for golfers (Henry et al., 2015; Lehman, 2006; Lephart, Smoliga, Myers, Sell, \& Tsai, 2007; Lindsay \& Horton, 2006). The main aim of many of these programmes is to increase strength, power, and flexibility in order to improve performance and reduce injury risk within the sport (Doan, Newton, Kwon, \& Kraemer, 2006; Keogh et al., 2009; Sell et al., 2007). Bull \& Bridge, (2012) found that, following an 8-week plyometric training intervention, X-Factor at the top of the backswing increased from $61.0 \pm 8.0^{\circ}$ to 68.0 $\pm 11.0^{\circ}$. Furthermore, this increase in the X-Factor enabled golfers to increase club head velocity by $4.7 \%$ and driving distance by $5.9 \%$ following the intervention. There is limited research regarding flexibility interventions and their relationship with the X-Factor and XFactor stretch; however, by improving flexibility, it is suggested that golfers are able to create 
faster club head speed, due to an increased range of motion in the backswing (Chu, Sell, \& Lephart, 2010; Draovitch \& Simpson, 2007).

Yoga has become a popular method of flexibility training in many sports, including golf (Briegel-Jones, Knowles, Eubank, Giannoulatos, \& Elliot, 2013). Yoga is suggested to improve muscular strength (Gothe, Kramer, \& Mcauley, 2014) and range of motion (Amin \& Goodman, 2014), which are all key components in performing the golf swing proficiently (Draovitch \& Simpson, 2007). Many yoga poses use body weight against gravity to exert force, which can improve muscular strength (Gothe et al., 2014). Golfers need a high level of muscular strength to achieve a powerful golf swing, especially within the trunk area, as this will affect a golfer's maximum thorax and club head velocities (Draovitch \& Simpson, 2007). Furthermore, it has been reported that regular yoga practice will improve an individual's range of motion (Amin \& Goodman, 2014), however, this is yet to be found in relation to the golf swing.

Therefore, the aim of the current study was to determine the effectiveness of a six-week yoga intervention with the aim of improving the X-Factor and X-Factor stretch during the golf swing. Furthermore, the current study aimed to examine the pelvis and torso rotation and lead hand velocity during the golf swing following the yoga intervention. It was hypothesized that performing a yoga training programme would improve golf swing mechanics and, therefore, increase the long game performance of the golfer.

\section{METHODS}

\section{Experimental Approach to the Problem}

In order to examine the given hypothesis of the current investigation, a randomized controlled pre and post-test experimental design was used. Two groups of single handicap golf players 
were randomized either into a six-week yoga intervention group or control group. This was to examine the effect of yoga training on the X-Factor, X-Factor stretch, pelvis and torso rotation and lead hand velocity during the golf swing.

\section{Participants}

Ten right-handed male amateur golfers volunteered to participate in this study. Five participants were randomized into an experiment group (mean \pm SD age: $19.60 \pm 2.30$ years; stature: $179.66 \pm 5.80 \mathrm{~cm}$; body mass: $87.04 \pm 17.86 \mathrm{~kg}$ and handicap: $5.00 \pm 3.00)$ and five participants into a control group (mean \pm SD age: $25.20 \pm 5.02$ years; stature: $184.02 \pm 4.80$ cm; body mass: $83.89 \pm 14.99 \mathrm{~kg}$ and handicap: $5.20 \pm 4.71$ ). Additional anthropometric measurements (shoulder offset, elbow width, wrist width, hand thickness, leg length, knee width, ankle width) were recorded during the initial stages of the testing process in order to run a customized Golf Model (Sorbie et al., 2018). Low handicap golfers were selected to participate in the study as it provided a representative sample of the population, whilst excluding the probability of technique having a major influence on results (Joyce, Burnett, Cochrane, \& Reyes, 2016). Participants had to be free of any musculoskeletal injuries for a period of three months prior to the study, as these factors may limit flexibility and golf swing attributes (Lindsay \& Horton, 2006). Participants were also required to undertake no conditioning or resistance training 48 hours prior to the testing sessions. All participants completed a consent form and physical readiness questionnaire before participating in the current study. Full ethical approval was granted from Abertay University, School of Health Sciences prior to data collection. 


\section{Experimental Procedure}

All participants performed a standardized warm up, which consisted of a moderate paced run 150 for 2.5 minutes. Participants then performed 10 body weight squats, 10 keyhole arm swings and 10 air swings prior to data collection. Following the warm-up, participants performed 5 golf swings using a standardized driver. All golf shots were struck from a rubber tee which was placed on an artificial golf mat fixed to the floor in the centre of the laboratory. During each golf shot, participants were instructed to perform their standard golf swing with the aim of maximizing distance and accuracy. Participants were instructed to aim towards a target area on the wall.

Following the initial test, participants that were assigned to the experiment group took part in a six-week yoga intervention, targeting key areas of inflexibility within golfers including the shoulders, torso and pelvis. Participants performed six 30-minute sessions over the six-week intervention period (Amin \& Goodman, 2014). Participants in the control group were instructed completing the identical protocol completed on the first visit to the laboratory.

\section{Data Collection}

During the 5 golf shots that were performed before and after the six-week intervention, threedimensional (3-D) data were collected using the an 8-camera Vicon Bonita (Oxford Metrics Ltd, United Kingdom) Motion Analysis System operating at $200 \mathrm{~Hz}$. To ensure the system had been correctly calibrated, the camera residual was $<0.2 \mathrm{~mm}$. Spherical retro-reflective markers $(1.4 \mathrm{~cm})$ were adhered to the skin on anatomical regions according to the adapted version of the Vicon Plug-in-Gait Model (Vicon Motion Systems Ltd, Oxford, UK) (Sorbie et al., 2018) 
using double-sided tape. To ensure accurate 3-D data collection, participants were asked to wear tight fitting shorts and their own golf shoes when performing all golf shots.

\section{Data Reduction and Analysis}

Following the labelling of all trials, the data was smoothed using a Butterworth filter $(15 \mathrm{~Hz})$ and exported to Microsoft Excel (version 2013). A multi-segment model used to analyse the X-Factor in the current study was developed using BodyBuilder (Oxford, UK) and used in Vicon version 2.7. This multi-segment model, which calculates the torso and pelvic separation, is suggested as a valid method of obtaining X-Factor values. A schematic representation of the model used in the current study has been previously published (Brown et al., 2013). The XFactor stretch was calculated by subtracting the X-Factor at the top backswing from the maximum X-Factor value during the downswing. Other swing phases of interest included: lead arm parallel to the ground during the downswing, pre-impact (the point at the last $40 \mathrm{~ms}$ before impact), and impact (Figure 1) (Myers et al., 2008). These positions were determined from the position of the retro-reflective markers on the upper extremity (Myers et al., 2008). In order to measure the lead hand velocity (left marker (LFIN)), the frame at the lead arm parallel during the downswing, pre-impact and impact of the ball was identified. Following this, the XYZ data was calculated using the following equation:

$$
\text { Absolute position }=\sqrt{x^{2}+y^{2}+z^{2}}
$$

***INSERT FIGURE 1 NEAR HERE***

\section{Statistical Analysis}

All calculations were performed on Microsoft Excel (version 2013) and SPSS (version 23). Normal distribution for all variables was assessed using the Shapiro-Wilk test. A null 
hypothesis for the tests was accepted due to all $\mathrm{P}$ values being greater than 0.05 . Upon this being determined, an unpaired T-Test was used to identify differences in swing mechanics data sets when measuring the effects of the yoga intervention. The unpaired T-Test measured the absolute differences between the pre and post measures and was selected due to the small sample size used within this feasibility study. $\mathrm{P} \leq 0.05$ was considered significant. In addition to the P value, effect sizes were calculated using Cohen's d method (Cohen, 1988). The following scale of thresholds was used to analyse the magnitudes of effect size: 0.80 large; 0.50 medium; and 0.20 small. Clinically meaningful data was calculated based on a previously used method (Liow \& Hopkins, 2003), which was interpreted qualitatively as follows: $<1 \%$, almost certainly not; $<5 \%$, very unlikely; $<25 \%$, unlikely; $25-75 \%$, possible; $>75 \%$, likely; $>95 \%$, very likely; and $>99 \%$ almost certain.

\section{RESULTS}

\section{X-Factor}

Significant differences were observed for the X-Factor at the top of the backswing following the completion of the yoga intention $(\mathrm{P} \leq 0.05)$ and a medium effect between groups was observed $(d=0.50)$. From a qualitative perspective, these results are very likely $(96 \%)$ to be clinically meaningful (Figure 2).

\section{***INSERT FIGURE 2 NEAR HERE***}

\section{X-Factor Stretch}

No significant differences were observed for the X-Factor stretch during the golf swing following the completion of the yoga intention $(\mathrm{P}=0.21)$. A medium effect was observed 
222 between groups $(\mathrm{d}=0.57)$, however, these results are unlikely (30\%) to be clinically

223

224

225

226

227

228

229

230

231

232

233

234

235

236

237 meaningful (Figure 3).

\section{***INSERT FIGURE 3 NEAR HERE***}

\section{Swing Mechanics}

The means, standard deviations, group statistical differences, group effect sizes and qualitative chances for each of the variables assessed are displayed in Table 1. Specifically, angle of rotations of the pelvis and torso for the four phases of the golf swing are displayed in Table 1. In addition to the angles of rotation, hand velocities at the three latter stages of the golf swing are also displayed in Table 1.

***INSERT TABLE 1 NEAR HERE***

\section{DISCUSSION}

The aim of the current study was to evaluate the effectiveness of a six-week yoga training programme on golf swing mechanics. Specifically, the study aimed to describe the changes, if any, in the X-Factor and X-Factor stretch during the golf swings performed pre and post the six-week yoga intervention. Furthermore, the current study aimed to investigate the changes, if any, in pelvis and torso rotation and lead hand velocities during specific phases of the golf swing following the yoga intervention. It was hypothesized that swing mechanics when performing golf swings with the driver would improve following the yoga intervention due to the increased ROM. Additionally, due to this hypothesized increased ROM, it was hypothesized that lead hand velocity would increase throughout the latter phases of the golf swing. As a result of the findings of this feasibility study, the hypotheses in relation to ROM were partially accepted. Moreover, the hypotheses for lead hand velocity during the golf swing 
were rejected. However, as discussed later in this section, these results have been rejected with caution.

As previously discussed, scientific literature often describes the separation between the pelvis and torso at the top of the backswing as the X-Factor (Mclean \& Andrisani, 1996). Whereas the X-Factor stretch refers to the separation of the pelvis and torso during the initial stages of the downswing (Mclean \& Andrisani, 1996). From a biomechanics perspective, the correct pelvic and torso rotation throughout the golf swing is essential for maximising club head speed which, in turn, improves driving performance (Joyce, 2016). It has been previously reported that as the skill level of the golfer increases (lower handicap), pelvis rotation reduces at the top of the backswing and then increases during the downswing and impact phases (Myers et al., 2008). These researchers also reported that upper torso rotation during the four phases of the golf swing were similar when comparing skill level. Although the upper torso levels were similar between the different levels, the reduction in pelvic rotation resulted in greater upper torso-pelvic separation (Myers et al., 2008). It has been previously reported that the separation of the torso and pelvis requires a good level of flexibility and ROM, and is often attributed to lower handicap golfers (Lephart et al., 2007).

The current study reported that several of the ROM measurements throughout the swing significantly increased following the six-week yoga intervention. Specifically, the X-Factor at the top of the backswing significantly increased $(\mathrm{P} \leq 0.05)$ and a medium effect $(d \geq 0.50)$ was observed when comparing the yoga intervention group and control group. As a result of a qualitative analysis, these results are very likely to be clinically meaningful, which suggests that the yoga intervention had a positive effect on the X-Factor. A similar medium effect $(d \geq$ 0.50) was observed within the $X-$ Factor stretch, however no statistical significance $(P \geq 0.05)$ 
was observed between groups. As a result of a qualitative analysis, these results are very unlikely to be clinically meaningful which suggests that the yoga intervention had no effect on the $\mathrm{X}$-Factor stretch. In relation to pelvis rotation, statistical significance $(\mathrm{P} \leq 0.05)$ was displayed at all phases of the golf swing with the exception of the impact of the ball $(\mathrm{P} \geq 0.05)$. During the phases that were significant between groups, medium $(d \geq 0.50)$ and large $(d \geq 0.80)$ interactions were also displayed. These results in relation to the findings are either very likely or likely to be clinically meaningful (Table 1). For the torso rotation, no significant differences were observed at any phases of the golf swing when comparing groups $(\mathrm{P} \geq 0.05)$. As a result of the effect size measurements and qualitative analysis (Table 1), it was deemed that the sixweek yoga intervention is unlikely to influence torso rotation during the golf swing. These findings enabled the experimental hypothesis in relation to ROM to be partially accepted. Finally, no significant difference $(\mathrm{P} \geq 0.05)$ was observed in lead hand velocity at any phases of the golf swing, however, a medium effect $(d \geq 0.50)$ was found at impact. As a result of these findings, in relation to lead hand velocity the second experimental hypothesis was rejected. However, this rejection is reported with caution due to the varying qualitative results (Table 1). These inconsistencies may be due to the small sample size used within this feasibility study.

The current findings in relation to the X-Factor at the top of the backswing and torso-pelvis separation are in agreement with previous research that has investigated flexibility and ROM interventions (Amin \& Goodman, 2014; Lephart et al., 2007). Specific to golf, Lephart et al., (2007) reported a $6.8 \%$ increase $\left(49.8^{\circ} \pm 7.6\right.$ vs. $\left.53.5^{\circ} \pm 5.6\right)$ in the $X$-Factor following an eight-week stretching programme which is similar to the $9.72 \%$ increase $\left(51.9^{\circ} \pm 8.7\right.$ vs. 57.2 ${ }^{\circ} \pm 5.9$ ) observed in the current study. This increase in X-Factor displayed within the current study and the study by Lephart and colleagues is positive in relation to golf performance. It has 
been previously reported that an increase in X-Factor is correlated with skill level (Myers et al., 2008) and ball velocity (Chu et al., 2010). In relation to the pelvis rotation at the top of the backswing, Lephart et al., (2007) reported a $13.4 \%$ reduction $\left(-56.1^{\circ} \pm 10.8\right.$ vs. $\left.-49.4^{\circ} \pm 6.8\right)$ in pelvic rotation following the intervention. The current study reported a $7.7 \%$ reduction ($56.4^{\circ} \pm 10.6$ vs. $-52.4^{\circ} \pm 9.9$ ) following the yoga intervention. In relation to golf performance, it has been reported that golfers that displayed reduced pelvis rotation at the top of the backswing also displayed greater carry distance (Lephart et al., 2007), ball velocity (Lephart et al., 2007; Myers et al., 2008) and club head velocity (Lephart et al., 2007). Subsequently, as the golf swing progresses to the downswing and impact phases, pelvis rotation increases when examining golfers with a greater ball velocity (Myers et al., 2008). These results are similar to the current study, where pelvic rotation increased during the downswing, pre-impact and impact phases of the golf swing following the six-week yoga intervention. Although the current study did not measure performance variables in relation to the golf club or golf ball, lead hand velocity was measured. Following the yoga intervention, lead hand velocity increased during the acceleration phase (6.9\%), pre-impact phase (10.2\%) and impact phase (5.9\%). Although these increases appear to be positive in relation to golf performance, no significant changes were observed following the yoga intervention. However, as a result of the qualitative analysis, the lead arm results at the acceleration phase and pre-impact phase are likely to be clinically meaningful. These inconsistencies within the results of the current study and with the literature could also be due to the low sample size within the current study.

In relation to the torso rotation at the top of the backswing, Lephart et al., (2007) reported a $3.8 \%$ reduction $\left(-106.4^{\circ} \pm 9.5\right.$ vs. $\left.-102.6^{\circ} \pm 8.1\right)$, however, these changes were not significant. Similar to these results, the current study reported no significant changes and only small interactions were observed following the six-week yoga intervention $\left(-108.34^{\circ} \pm 15.4\right.$ vs. - 
$\left.108.57^{\circ} \pm 10.5\right)$. Although the torso rotation at the top of the backswing was not affected following the six-week yoga intervention, the decrease in pelvis rotation was greater than the change in torso rotation, which significantly increased the X-factor. Therefore, these findings suggest that a more stable torso will enable a greater torso-pelvic separation, resulting in improved swing mechanics (Lephart et al., 2007; Myers et al., 2008). These findings during the backswing were also consistent for the downswing, pre-impact and impact phases of the golf swing (Table 1).

Although there are no previous scientific studies that have investigated the effect of yoga on golf swing mechanics, previous research has investigated the effect of yoga to increase ROM and flexibility. Amin \& Goodman, (2014) reported that flexibility significantly improved following a six-week yoga intervention. Specifically, these researchers reported significant increases in the sit and reach test $(29.50 \mathrm{~cm} \pm 7.08 \mathrm{vs} .30 .87 \mathrm{~cm} \pm 7.01)$ following a 6-week yoga programme. Although this study did not measure specific movements that are relevant to the golf swing, the two studies show that performing yoga over a six-week period can be an effective method to increase ROM and, therefore, can be a useful method when aiming to increase ROM during the golf swing.

Due to the positive findings in relation to yoga and golf movement mechanics within this feasibility study, a full-scale trial is warranted. Therefore, as a result of the small sample size within this feasibility study, the current findings must be interpreted with caution as they may not be generalizable to a larger population. Furthermore, only low handicap golfers were tested in this feasibility study, therefore, the result may only be valid for this population. Although this skill level may be viewed as a limitation, technique variations within higher skilled golfers is reduced, which can be more beneficial when testing with a smaller sample size. As the 
current study was feasibility of the effect of yoga on golf swing mechanics, future studies should incorporate a larger sample size, include females within the sample and also include a greater variation of golfers. Furthermore, no performance variables in relation to the golf driver or the golf ball were measured during the testing of this study, only hand velocities were measured. Future studies examining the effect of a yoga intervention on golf performance should investigate performance variables such as club head speed, ball speed and carry distance in order to truly reflect performance.

\section{CONCLUSION}

The results of this feasibility study indicate that yoga may be a promising intervention in order to improve golf swing mechanics, with the aim of improving long game performance. Specifically, yoga training improves torso-pelvic separation which, in turn, can improve long game performance in golf. This feasibility study can inform future research designs and fullscale studies where researchers want to measure the effect of yoga on golf swing mechanics and golf performance variables. From the current findings, applied practitioners may wish to explore yoga as a training method in order to improve golf movement patterns, however, future research is required to confirm the effect of the use of yoga within the game of golf, with the aim of improving golf swing mechanics and golf performance variables.

\section{REFERENCES}

Amin, D. J., \& Goodman, M. (2014). The effects of selected asanas in Iyengar yoga on flexibility: Pilot study. Journal of Bodywork and Movement Therapies, 18(3), 399-404. http://doi.org/10.1016/j.jbmt.2013.11.008

An, J., Wulf, G., \& Kim, S. (2013). Increased carry distance and X-Factor stretch in golf through an external focus of attention. Journal of Motor Learning and Development, 1 , $2-11$.

Briegel-Jones, R. M. H., Knowles, Z., Eubank, M. R., Giannoulatos, K., \& Elliot, D. (2013). A Preliminary Investigation into the Effect of Yoga Practice on Mindfulness and Flow 
in Elite Youth Swimmers. The Sport Psychologist, 27(4), 349-359.

http://doi.org/10.1123/tsp.27.4.349

Brown, S. J., Selbie, W. S., \& Wallace, E. S. (2013). The X-Factor: an evaluation of common methods used to analyse major inter-segment kinematics during the golf swing. Journal of Sports Science, 31, 1156-1163.

Bull, M., \& Bridge, M. W. (2012). The Effect of an 8-Week Plyometric Exercise Program on Golf Swing Kinematics. International Journal of Golf Science, 1, 42-53. http://doi.org/10.1017/CBO9781107415324.004

Cheetham, P. J., Martin, P. E., Mottram, R. E., \& St. Laurent, B. F. (2001). The importance of stretching the "X-Factor" in the downswing of golf: The "X-Factor Stretch." Optimising Performance in Golf, 192-199. http://doi.org/10.1039/b106052j

Chettle, D. K., \& Neal, R. J. (2001). Optimising performance in golf. In A. A. P. Ltd. (Ed.), Strength and conditioning for golf. (pp. 207-223). Brisbane.

Chu, Y., Sell, T. C., \& Lephart, S. M. (2010). The relationship between biomechanical variables and driving performance during the golf swing. Journal of Sports Sciences, 28(11), 1251-1259. http://doi.org/10.1080/02640414.2010.507249

Cohen, J. (1988). Statistical power analysis for the behavioral sciences. Hillsdale (2nd ed.). Erlbaum.

Cole, M. H., \& Grimshaw, P. N. (2008a). Electromyography of the trunk and abdominal muscles in golfers with and without low back pain. Journal of Science and Medicine in Sport, 11(2), 174-181. http://doi.org/10.1016/j.jsams.2007.02.006

Cole, M. H., \& Grimshaw, P. N. (2008b). Trunk muscle onset and cessation in golfers with and without low back pain. Journal of Biomechanics, 41(13), 2829-2833. http://doi.org/10.1016/j.jbiomech.2008.07.004

Cools, A. M., Johansson, F. R., Cambier, D. C., Velde, A. Vande, Palmans, T., \& Witvrouw, E. E. (2010). Descriptive profile of scapulothoracic position, strength and flexibility variables in adolescent elite tennis players. British Journal of Sports Medicine, 44(9), 678-684. http://doi.org/10.1136/bjsm.2009.070128

Doan, B. K., Newton, R. U., Kwon, Y.-H., \& Kraemer, W. J. (2006). Effects of physical conditioning on intercollegiate golfer performance. Journal of Strength and Conditioning Research, 20(1), 62-72. http://doi.org/10.1519/R-17725.1

Draovitch, R., \& Simpson, P. (2007). Complete Conditioning for Golf. Leeds: Human Kinetics.

Farrally, M. R., Cochran, A. J., Crews, D. J., Hurdzan, M. J., Price, R. J., Snow, J. T., \& Thomas, P. R. (2003). Golf science research at the beginning of the twenty-first century. Journal of Sports Sciences, 21, 753-765. http://doi.org/10.1080/0264041031000102123

Gothe, N. P., Kramer, A. F., \& Mcauley, E. (2014). The effects of an 8-week hatha yoga intervention on executive function in older adults. Journals of Gerontology - Series A Biological Sciences and Medical Sciences, 69(9), 1109-1116. http://doi.org/10.1093/gerona/glu095

Hellström, J. (2009). Competitive Elite Golf. Sport. Med., 39(9), 723-741. http://doi.org/10.2165/11315200-000000000-00000

Henry, E., Berglund, K., Millar, L., \& Locke, F. (2015). Immediate effects of a dynamic rotation-specific warm-up on $\mathrm{x}$-factor and $\mathrm{x}$-factor stretch in the amateur golfer. The International Journal of Sports Physical Therapy, 10(7), 998-1007. 
Joyce, C. (2016). An examination of the correlation amongst trunk flexibility, $x$-factor and clubhead speed in skilled golfers. Journal of Sports Sciences, 1-7. http://doi.org/10.1080/02640414.2016.1252052

Joyce, C., Burnett, A., Cochrane, J., \& Reyes, A. (2016). A preliminary investigation of trunk and wrist kinematics when using drivers with different shaft properties. Sports Biomechanics, 15, 61-75.

Keogh, J.W., \& Hume, P. (2012). Evidence for biomechanics and motor learning research improving golf performance. Sports Biomechanics, 11(2), 288-309. http://doi.org/10.1080/14763141.2012.671354

Keogh, J.W., Marnewick, M. C., Maulder, P. S., Nortje, J. P., Hume, P. A., \& Bradshaw, E. J. (2009). Are anthropometric, flexibility, muscular strength, and endurance variables related to clubhead velocity in low- and high-handicap golfers? Journal of Strength and Conditioning Research, 23(6), 1841-1850. http://doi.org/10.1519/JSC.0b013e3181b73cb3

Lehman, G. (2006). Resistance training for performance and injury prevention in golf. The Journal of the Canadian Chiropractic Association, 50(1), 27-42.

Lephart, S., Smoliga, J., Myers, J., Sell, T., \& Tsai, Y. (2007). An eight-week golf-stretching exercise program improves physical characteristics, swing mechanics, and golf performance in recreational golfers. Journal of Strength and Conditioning Research, 21(3), 860-869. http://doi.org/10.1519/R-20606.1

Lindsay, D. M., \& Horton, J. F. (2006). Trunk rotation strength and endurance in healthy normals and elite golfers with and without lower back pain. North American Journal of Sports Physical Therapy, 1(2), 80-89.

Liow, D. K., \& Hopkins, W. G. (2003). Velocity specificity of weight training for Kayak Sprint performance. Medicine and Science in Sports and Exercise, 35(7), 1232-1237. http://doi.org/10.1249/01.MSS.0000074450.97188.CF

Mclean, J., \& Andrisani, J. (1996). The X-factor swing: And other secrets to power and distance. New York: Harper Collins.

Myers, J., Lephart, S., Tsai, Y.-S., Sell, T., Smoliga, J., \& Jolly, J. (2008). The role of upper torso and pelvis rotation in driving performance during the golf swing. Journal of Sports Sciences, 26(2), 181-188. http://doi.org/10.1080/02640410701373543

Sell, T. C., Tsai, Y. S., Smoliga, J. M., Myers, J. B., \& Lephart, S. M. (2007). Strength, flexibility, and balance characteristics of highly proficient golfers. Journal of Strength and Conditioning Research, 21(4), 1166-1171. http://doi.org/10.1519/R-21826.1

Sorbie, G. G., Gu, Y., Baker, J. S., \& Ugbolue, U. C. (2018). Analysis of the X-Factor and XFactor stretch during the completion of a golf practice session in low-handicap golfers. International Journal of Sports Science \& Coaching, O(0), 1-7. http://doi.org/10.1177/1747954118791330

Tilley, N. R., \& Macfarlane, A. (2012). Effects of different warm-up programs on golf performance in elite male golfers. The International Journal Fo Sports Physical Therapy, 7(4), 388-395.

Young, W., Clothier, P., Otago, L., \& Liddell, D. (2004). Acute effects of static stretching on hip flexor and quadriceps flexibility, range of motion and foot speed in kicking in football. Journal of Science and Medicine in Sport, 7(1), 23-31.

Zheng, N., Barrentine, S., Fleisig, G., \& Andrews, J. (2008). Kinematic analysis of swing in 


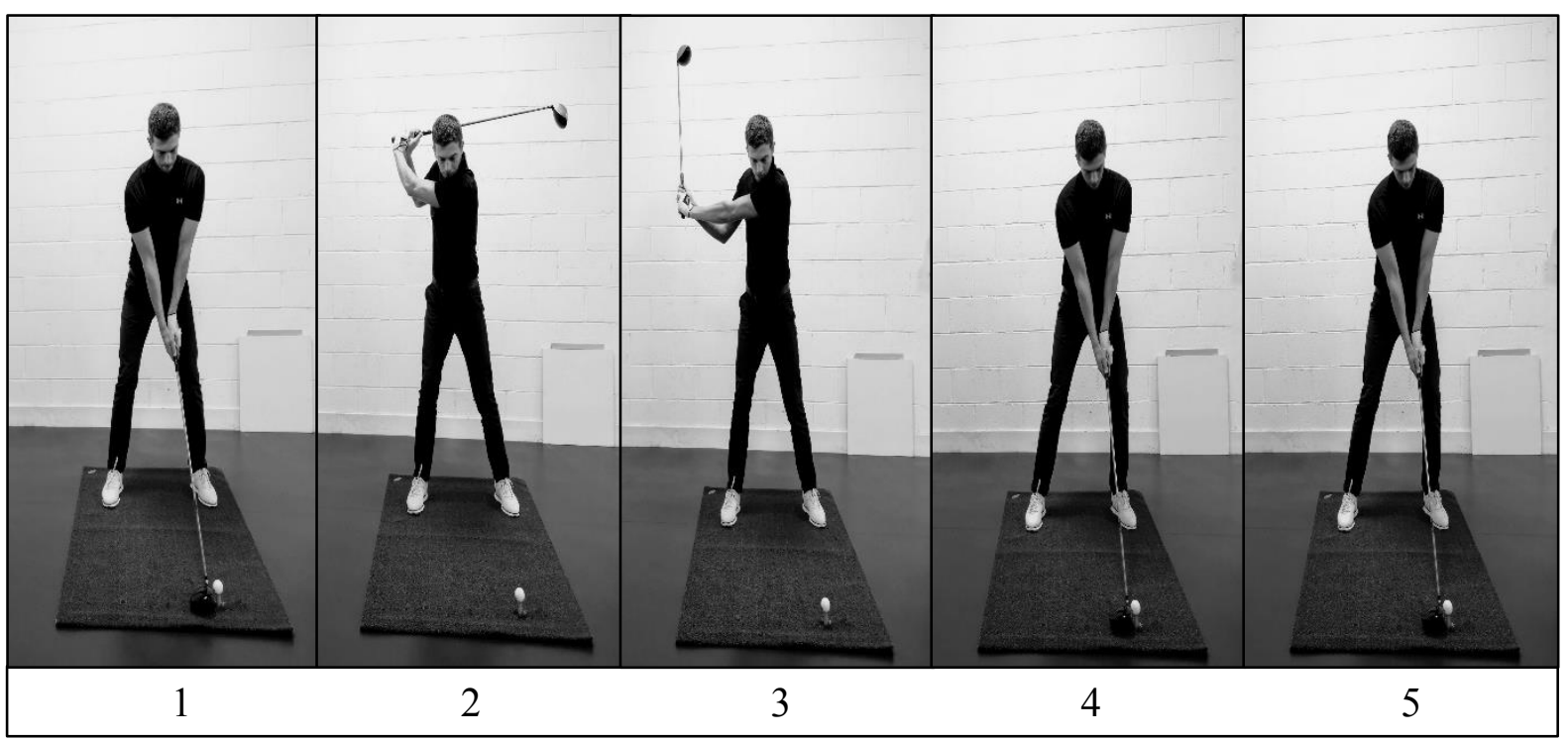

Figure 1: Silhouette description of the phases of the golf swing. 1 - Set-up position, 2 - Top of the backswing, 3 - Lead arm parallel to the ground during the downswing, 4 - Pre-impact (the point at the last $40 \mathrm{~ms}$ before impact), 5 - Impact of the golf ball. 


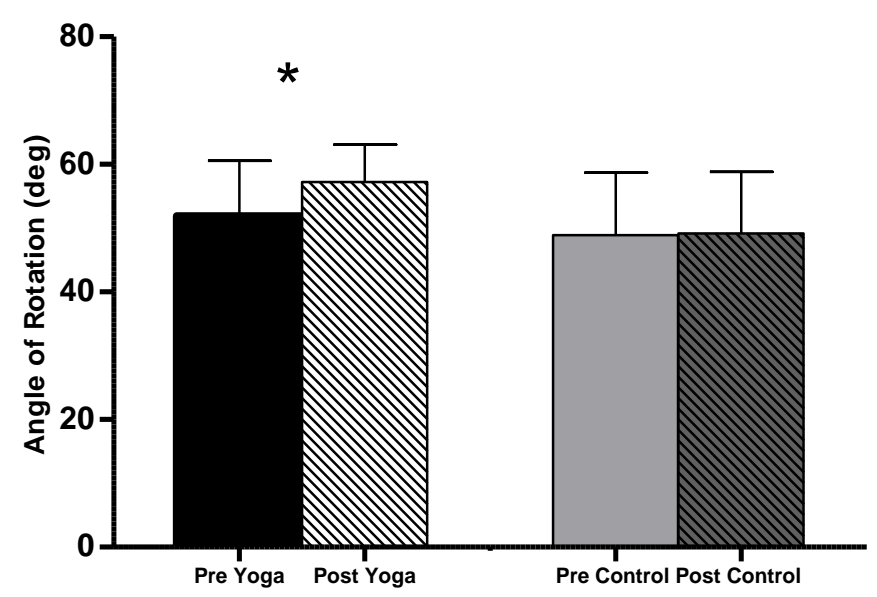

Figure 2: X-Factor at the top of the backswing for the yoga intervention group and the control group. Note: Statistical significance is shown with * $(\mathrm{P} \leq 0.05)$. 


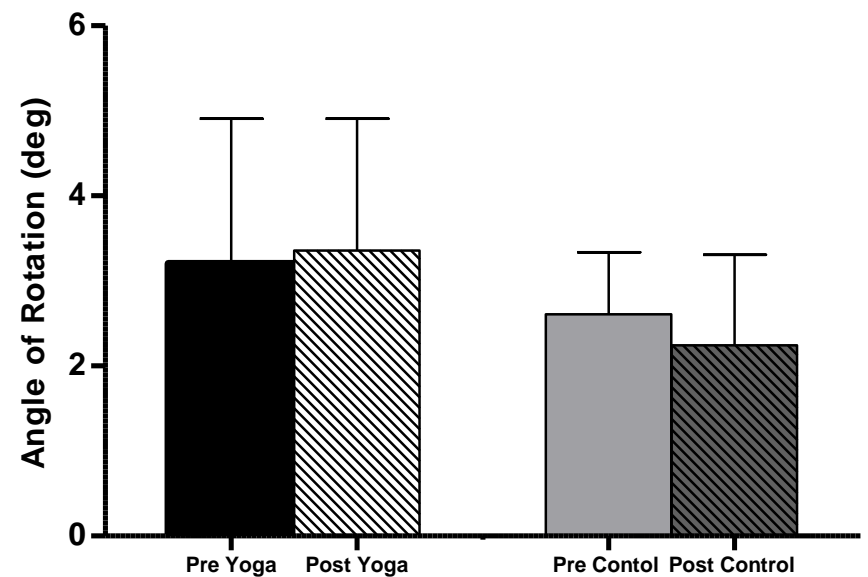

Figure 3: X-Factor stretch for the yoga intervention group and the control group. 
Table 1: The means, standard deviations, group statistical differences, and group effect sizes for swing mechanics

\begin{tabular}{|c|c|c|c|c|c|c|c|}
\hline Swing Mechanics & Pre Yoga & Post Yoga & Pre Control & Post Control & $\boldsymbol{P}$ & $d$ & Qualitative \\
\hline Pelvis Rotation at Top of Swing $\left(^{\circ}\right)$ & $-56.42 \pm 10.64$ & $-52.39 \pm 9.92$ & $-49.69 \pm 11.36$ & $-48.73 \pm 10.89$ & $0.01 *$ & $\underline{0.29}$ & $89 \%$ \\
\hline Pelvis Rotation at Parallel $\left({ }^{\circ}\right)$ & $5.79 \pm 2.15$ & $8.07 \pm 3.27$ & $1.66 \pm 5.32$ & $2.06 \pm 4.92$ & $0.04 *$ & $\underline{0.42}$ & $89 \%$ \\
\hline Pelvis Rotation at Pre-Impact $\left(^{\circ}\right)$ & $23.90 \pm 5.07$ & $26.91 \pm 5.05$ & $22.90 \pm 3.73$ & $23.16 \pm 3.82$ & $0.04 *$ & 0.69 & $95 \%$ \\
\hline Pelvis Rotation at Impact $\left(^{\circ}\right)$ & $33.56 \pm 3.20$ & $36.57 \pm 4.46$ & $32.57 \pm 5.49$ & $32.28 \pm 6.54$ & 0.09 & 0.86 & $92 \%$ \\
\hline Torso Rotation at Top of Swing $\left(^{\circ}\right)$ & $-108.34 \pm 15.40$ & $-108.57 \pm 10.45$ & $-98.55 \pm 11.68$ & $-97.87 \pm 10.78$ & 0.76 & 0.06 & $12 \%$ \\
\hline Torso Rotation at Parallel $\left(^{\circ}\right)$ & $-32.00 \pm 8.47$ & $-29.03 \pm 10.87$ & $-33.55 \pm 12.21$ & $-32.83 \pm 12.05$ & 0.15 & $\underline{0.25}$ & $\underline{63 \%}$ \\
\hline Torso Rotation at Pre-Impact $\left({ }^{\circ}\right)$ & $-10.28 \pm 4.83$ & $-8.04 \pm 4.70$ & $-6.19 \pm 10.89$ & $-5.28 \pm 11.46$ & 0.26 & 0.19 & $47 \%$ \\
\hline Torso Rotation at Impact $\left(^{\circ}\right)$ & $4.82 \pm 3.39$ & $6.46 \pm 2.40$ & $4.65 \pm 7.80$ & $4.67 \pm 8.87$ & 0.11 & $\underline{0.36}$ & $79 \%$ \\
\hline Hand Velocity at Parallel $\left(\mathrm{m} . \mathrm{s}^{-1}\right)$ & $30.62 \pm 3.58$ & $32.90 \pm 3.58$ & $28.29 \pm 6.04$ & $28.61 \pm 6.31$ & 0.18 & $\underline{0.44}$ & $79 \%$ \\
\hline Hand Velocity at Pre-Impact $\left(\mathrm{m} . \mathrm{s}^{-1}\right)$ & $27.17 \pm 2.77$ & $30.27 \pm 1.76$ & $24.61 \pm 5.67$ & $25.05 \pm 5.78$ & 0.17 & $\underline{0.62}$ & $84 \%$ \\
\hline Hand Velocity at Impact $\left(\mathrm{m} \cdot \mathrm{s}^{-1}\right)$ & $22.96 \pm 2.50$ & $24.39 \pm 2.51$ & $20.27 \pm 4.78$ & $20.59 \pm 4.42$ & 0.33 & $\underline{0.26}$ & $59 \%$ \\
\hline
\end{tabular}

Note: Statistical significance is shown with * $(\mathrm{P} \leq 0.05)$. Large effect $(d>0.80)$ between groups are shown in bold font. Medium effect $(d>0.50)$ between groups are shown in italic font. Small effect $(d>0.20)$ between groups are shown in underlined font. Qualitative chance: Very likely displayed in bold font. Likely displayed in italic font. Possibly displayed in underlined font. Parallel - lead arm parallel to the ground. 